\title{
Impact-Force Estimation by Quadratic Spline Approximation*
}

\author{
Fergyanto E. GUNAWAN $^{* *}$, Hiroomi HOMMA*** and Yuichi MORISAWA ${ }^{\dagger}$ \\ ** Department of Mechanical Engineering, Toyohashi University of Technology \\ 1-1 Hibarigaoka, Tempaku-cho, Toyohashi-shi, 441-8580, Japan \\ E-mail: gunawan @mech.tut.ac.jp \\ *** International Cooperation Center for Engineering Education Development \\ 1-1 Hibarigaoka, Tempaku-cho, Toyohashi-shi, 441-8580, Japan \\ E-mail: homma@icceed.tut.ac.jp \\ Department of Mechanical Engineering, Toyohashi University of Technology \\ 1-1 Hibarigaoka, Tempaku-cho, Toyohashi-shi, 441-8580, Japan
}

\begin{abstract}
This paper focuses on a solution of the ill-posed inverse problem of the impact-force reconstruction. The solution approximates the impact-force with a regularized quadratic spline having an uniform distribution of knots along the time domain of analysis. The approximation results in a small size, ill-posed, and rank-deficient system equation, which is then solved by the truncated singular value decomposition and L-curve methods. The proposal that is verified by three impact-force problems provides acceptable estimated impact-forces. However, the estimated impact-force in the unloading part rather oscillates in particular when the data are severely contaminated with the noise. Therefore, the present method needs to be further developed to allow a non-uniform knots distribution such that the regularization in the unloading part can be increased.
\end{abstract}

Key words : Inverse Problem, Regularization, Least-Squares, Impact-Force

\section{Introduction}

The inverse analysis for the force reconstruction, impact-force in particular, is necessary when the force is difficult to be directly measured by the conventional measurement method such as in impact of a small particle on a plate.

Although the force reconstruction by the inverse analysis is difficult in obtaining accurate solution, but since it has many potential applications, many researchers have proposed solution methods to overcome those difficulties.

Hansen ${ }^{(1)}$ has comprehensively presented the nature of the ill-posed problem in general, and has shown a number of solution methods, direct and iterative, on the basis of the Tikhonov regularization method ${ }^{(2)}$. Tikhonov regularization states that the best solution of the ill-posed problem is one that minimizes the least-square criterion and also minimizes the noise effect in the solution. The idea has been widely implemented for solving various force reconstruction problems: identifying the moving loads on a multi-span bridge ${ }^{(3)}$, identifying the impact-force on a simply supported beam ${ }^{(4)}$, and identifying the impact-force on a circular plate ${ }^{(5)}$. Not only in the time domain, the Tikhonov regularization has also been used in the frequency domain to identify the harmonic loads on a beam ${ }^{(6)}$.

However, for some impact-force inverse problems, the least-squares criterion, the first term of the Tikhonov regularization, is also able to provide a quite satisfactory solution. By using the criterion, Cardi et. al. ${ }^{(7)}$ successfully recovered the force acting on a ceramic body armor; Wang $^{(4)}$ reasonably estimates the impact-force magnitude by minimizing the difference between the measured modal response to a reference modal response.

Although many solution methods have been proposed, the accuracy of the impact-force 
inverse analysis can be further increased by taking into account priori data of the impact-force besides utilizing some sorts of the numerical regularizations. Those priori data are information that characterize the impact-force profile.

The impact-force inverse problem has uniquenesses in comparison with other inverse problems. The solution, the impact-force time history, usually contains two parts: the loading part and the unloading part. Typically, the loading part is a part where the force is initially zero, increases to its maximum, and then decreases to zero. In the unloading part, the force is constant at zero ${ }^{(8)}$. For such a problem, the widely used solution method of the singular value decomposition (SVD) method ${ }^{(1)}$ can easily approximate the loading part, but difficult to approximate the unloading part. It is because the SVD method approximates a solution by use a set of harmonic singular vectors. The unloading part has to be approximated by high frequency components of the singular vectors; unfortunately, those components are severely affected by the noise.

Therefore, we proposed to use the cubic basic spline to approximate the impact-force ${ }^{(9)}$. The approach allows us to take into account the solution priori information and provides good approximations for the loading and unloading parts. In the meantime, Dmitriev and Ingtem ${ }^{(10)}$ devised the regularized quadratic spline to solve the ill-posed problem. The method utilizes a stricter regularization. In this paper, we extend the method to cover the impact-force inverse problem. In addition, we also study their performance to solve a problem where the data are obtained from experiments. Such data usually contain rather big amount of noise; they may cripple a solution method although the method has been proved to provide a good solution on an analytical problem.

\section{Governing Equation for the Impact-Force Inverse Analysis}

An inhomogeneous Fredholm integral equation of the first kind is written as

$$
\int_{0}^{t} K(t, \tau) f(\tau) d \tau=e(t)
$$

and the problem is to find the function $f(\tau)$ for given the continuous kernel function $K(t, \tau)$, and the function $e(t)$.

For the impact problem in the linear elastic regime, the kernel function takes the form of the convolution function $h(t-\tau)$; therefore, Eq. (1) becomes

$$
\int_{0}^{t} h(t-\tau) f(\tau) d \tau=e(t)
$$

where $f(t)$ is the applied impact-force, $e(t)$ is the elastic response, and $h(t)$ denotes the impulse response function which is a complete characterization of the dynamic behavior of the structure $^{(11)}$. For the causal system, $h(t-\tau)=0$ for $t<\tau$. Using Riemann's sum, Eq. (2) can be expressed as

$$
\sum_{j=1}^{M} h\left(t_{i}-\tau_{j}\right) f\left(\tau_{j}\right) \Delta t=e\left(t_{i}\right), \quad i=1, \ldots, M,
$$

where $t_{i}=(i-1) \cdot \Delta t, \Delta t$ is the sampling time, and in the matrix-vector form is written as

$$
\mathbf{H} \mathbf{f}=\mathbf{e} .
$$

In Eq. (4), the system matrix $\mathbf{H}$ is a lower triangular matrix that has Toeplitz structure. In its first column lies the vector of the impulse response function.

\section{Quadratic Spline Approximation and Its Regularization}

\subsection{Quadratic Spline Approximation}

In this study, we approximate the impact-force $f(t)$ by a quadratic spline which is constructed by $N$ knots, and $N-1$ segments of the quadratic polynomial. Those knots are distributed uniformly at $t_{n}=(n-1) \cdot h$, where $n \in[1, N], h$ is $t_{n+1}-t_{n}$, and the final time of 
analysis is at $t_{N}=T$. Therefore, on $n$th segment, the impact force is expressed by a quadratic function of

$$
f(t)=f_{n+1} \frac{t-t_{n}}{h}+f_{n} \frac{t_{n+1}-t}{h}+\alpha_{n} \frac{\left(t-t_{n}\right)\left(t_{n+1}-t\right)}{h},
$$

for $t \in\left[t_{n}, t_{n+1}\right]$. The $f_{n}$ denotes the impact-force at time $t_{n}$.

Using the quadratic spline approximation, one can preserve up to the first derivative continuity of the function on each knot by forcing $\alpha_{n}$ to satisfy

$$
\alpha_{n+1}=-\alpha_{n}-\frac{f_{n}-2 f_{n+1}+f_{n+2}}{h} \text { for } n \in[1, N-2],
$$

or compactly written as

$$
\alpha_{m}=\sum_{k=1}^{N} \alpha_{m k} u_{k}
$$

which can also be expressed in $\alpha_{1}$ as

$$
\alpha_{n}=(-1)^{n-1} \alpha_{1}+\sum_{k=1}^{n-1}(-1)^{k} \frac{f_{n}-2 f_{n+1}+f_{n+2}}{h} .
$$

\subsection{Regularization}

Tikhonov regularization ${ }^{(2)}$ is the most commonly used method of regularization for solving ill-posed problems. The regularization determines the stable solution by minimizing the Euclidian norm of the impact force, $\|f(t)\|_{2}^{2}$, in addition to minimizing the residual norm. The use of the quadratic spline approximation allows us to impose stricter regularization. Instead of minimizing $\|f(t)\|_{2}^{2}$, we minimize $\|d f(t) / d t\|_{2}^{2}$ :

$$
\min _{\alpha_{n}} \sum_{n=1}^{N-1} \int_{t_{n}}^{t_{n+1}}\left(\frac{f_{n+1}-f_{n}}{h}+\alpha_{n} \frac{f_{n+1}+f_{n}-2 t}{h}\right)^{2} d t
$$

Since $\alpha_{n}$ can be compactly expressed in term of $\alpha_{1}$ as in Eq. (8), Eq. (9) can also be minimized with respect of $\alpha_{1}$.

Minimizing Eq. (9) with respect to $\alpha_{1}$, besides the fact that $d \alpha_{n} / d \alpha_{1}=(-1)^{n-1}$ (see Eq. (8)), gives

$$
\sum_{n=1}^{N-1}(-1)^{n-1} \int_{t_{n}}^{t_{n-1}}\left(\frac{f_{n+1}-f_{n}}{h}+\alpha_{n} \frac{f_{n+1}+f_{n}-2 t}{h}\right)\left(\frac{t_{n+1}+t_{n}-2 t}{h}\right) d t=0 .
$$

Substituting Eq. (8) into Eq. (10) leads us to

$$
\alpha_{1}=\frac{1}{b-a} \sum_{n=2}^{N-1}(-1)^{n} \sum_{k=1}^{n-1}(-1)^{k}\left(f_{k}-2 f_{k+1}+f_{k+2}\right) \text {. }
$$

Equations (5), (8), and (11) completely define the regularized impact-force profile in term of the quadratic spline. Since the solution minimizes $\|d f(t) / d t\|_{2}^{2}$, it also satisfies the Tikhonov regularization.

\subsection{Quadratic Spline Regularization for Solution of the Convolution Integral Equation}

We consider a solution of the Fredholm integral equation using the quadratic spline approximation. Substituting Eq. (5) into Eq. (1) gives

$$
\sum_{m=1}^{N-1} \int_{t_{m}}^{t_{m+1}} K(t, \tau)\left(f_{m+1} \frac{\tau-\tau_{m}}{h}+f_{m} \frac{\tau_{m+1}-\tau}{h}+\alpha_{m} \frac{\left(\tau-\tau_{m}\right)\left(\tau_{m+1}-\tau\right)}{h}\right) d \tau=e(t) .
$$

To obtain a compact expression of Eq. (12), we introduce $\beta_{m n}, \theta_{m n}$, and $\gamma_{m n}$, which are defined as:

$$
\beta_{m n}=\frac{1}{h} \int_{\tau_{m}}^{\tau_{m+1}} K\left(t_{n}, \tau\right)\left(\tau-\tau_{m}\right) d \tau
$$




$$
\theta_{m n}=\frac{1}{h} \int_{\tau_{m}}^{\tau_{m+1}} K\left(t_{n}, \tau\right)\left(\tau_{m+1}-\tau\right) d \tau
$$

and

$$
\gamma_{m n}=\frac{1}{h} \int_{\tau_{m}}^{\tau_{m+1}} K\left(t_{n}, \tau\right)\left(\tau-\tau_{m}\right)\left(\tau_{m+1}-\tau\right) d \tau
$$

Therefore, we reduce Eq. (12) to a compact linear system equation of

$$
\sum_{m=1}^{N}\left(f_{m+1} \beta_{m n}+f_{m} \theta_{m n}+\alpha_{m} \gamma_{m n}\right)=e\left(t_{n}\right)
$$

Equations (13) to (15) can also be expressed in term of the local moments:

$$
\begin{aligned}
\delta_{m n} & =\int_{0}^{h} K\left(t_{n}, \tau_{m}+\xi\right) d \xi, \\
\beta_{m n} & =\frac{1}{h} \int_{0}^{h} K\left(t_{n}, \tau_{m}+\xi\right) \xi d \xi,
\end{aligned}
$$

and

$$
\varphi_{m n}=\frac{1}{h} \int_{0}^{h} K\left(t_{n}, \tau_{m}+\xi\right) \xi^{2} d \xi
$$

As a consequence, we have

$$
\theta_{m n}=\delta_{m n}-\beta_{m n},
$$

and

$$
\gamma_{m n}=h \beta_{m n}-\varphi_{m n}
$$

Equation (16) in the local moment terms and with substitution of $\alpha_{m}$ by Eq. (7) is

$$
\begin{aligned}
& f_{1}\left(\left(\delta_{1 n}-\beta_{1 n}\right)+\sum_{m=1}^{N-1} \alpha_{m 1}\left(h \beta_{m n}-\varphi_{m n}\right)\right)+f_{N}\left(\beta_{N-1 n}+\sum_{m=1}^{N-1} \alpha_{m N}\left(h \beta_{m n}-\varphi_{m n}\right)\right) \\
& +\sum_{m=2}^{N-1} f_{m}\left(\beta_{N-1 n}+\left(\delta_{m n}-\beta_{m n}\right)\right)+\sum_{k=2}^{N-1} f_{k}\left(\sum_{m=1}^{N-1} \alpha_{m k}\left(h \beta_{m n}-\varphi_{m n}\right)\right)=e_{n}
\end{aligned}
$$

which should be solved to obtain $f_{n}$ at the knots.

\subsection{Truncated Singular Value Decomposition}

Although the regularization has been invoked in the quadratic spline in Eq. (22), the use of too many knots will naturally increase the solution fluctuation; therefore, a sort of regularization is necessary. For such a case, we suggest to use the truncated singular value decomposition method, presented in detail in Hansen ${ }^{(1)}$, instead of the Tikhonov regularization method. It is because the TSVD method, in our experience, usually produces a much more conservative solution. The truncated SVD solution can be obtained by

$$
f=\sum_{i=1}^{k} \frac{u_{i}^{T} e}{\sigma_{i}} v_{i},
$$

where $u_{i}$ is the left singular vector, $\sigma_{i}$ is the singular value, and $v_{i}$ is the right singular vector. The regularization parameter $k$ should be optimally selected, for an example, by use of the L-curve ${ }^{(1)}$. 


\section{Verifications}

\subsection{Single Degree of Freedom System}

In this section, we reconstruct the impact-force acting on the single degree of freedom (SDOF) system. Although the SDOF system response does not completely describe the impact response of the continuous structure, but in a narrow frequency range, the SDOF system provides a good approximation ${ }^{(12)}$. In addition, from mathematical stand point, the system is quite ill-posed with a condition number of $1 \times 10^{18}$.

The SDOF system consists of a mass $m$, a spring $k$, and a dashpot $\xi$. The impulse response function of the SDOF system can be expressed as

$$
h(t)=\frac{1}{m \omega_{d}} e^{-\xi \omega_{n} t} \sin \left(\omega_{d} t\right),
$$

where $\omega_{n}$ and $\omega_{d}$ denote the circular natural and circular damped frequencies. The both parameters can be computed by

$$
\omega_{n}=\sqrt{k / m},
$$

and

$$
\omega_{d}=\omega_{n} \sqrt{1-\xi^{2}} .
$$

In the present study, the following data are selected: $m=1 \mathrm{~kg}, k=1.0 \mathrm{E}+11 \mathrm{~N} / \mathrm{m}, \xi=0.1$, and the final time is $100 \mu \mathrm{s}$.

In addition to the impulse response function, the elastic response data are also necessary for the inverse analysis, and are produced by convolving the response function with an halfsine impact-force having a loading duration of $20 \mu$ s. Then, a small amount of the uniformly distributed pseudo-random noise having variance of $2 \%$ of the maximum elastic response data is superimposed to the data.

To demonstrate the ill-posed nature of the present problem, firstly, we reconstruct the impact-force by use the Gauss elimination with any regularization. The result that is shown in Fig. 1 is a typical direct solution of an ill-posed problem. To obtain an acceptable solution, regularization, for an example: by forcing certain degree of smoothness, is necessary.

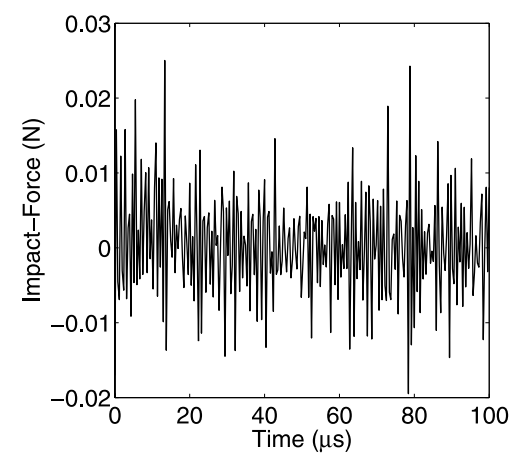

Fig. 1 The estimated impact-force without regularization.

The regularized solution obtained by the quadratic spline approximation and the truncated SVD method is shown in Fig. 2. To access the solution accuracy, we utilize a scalar parameter of the relative estimation error, $\Delta$, that is defined as

$$
\Delta=\frac{\left\|f_{e}-f_{e s t}\right\|_{2}}{\left\|f_{e}\right\|_{2}} \times 100,
$$

where $f_{e}$ is the vector of the exact solution and $f_{e s t}$ is the vector of the estimated solution. The estimated solution obtained in Fig. 2 has the relative estimation error of $4.7 \%$.

The above solution is obtained by use 64 knots that distributed uniformly along the time span of $100 \mu$ s. Using that number of knots, the system matrix will be in size of $64 \times 64$ 


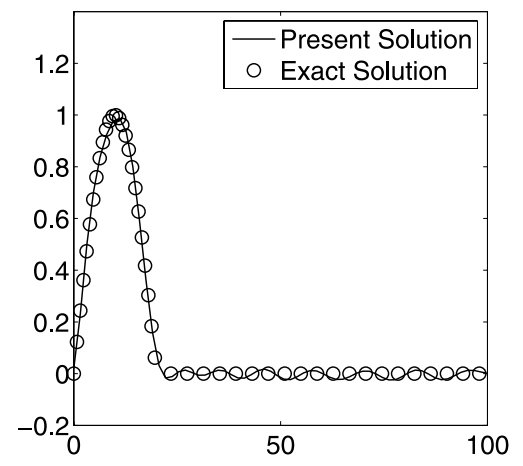

Fig. 2 The estimated impact-force with the quadratic spline regularization for the SDOF system.

where it can be decomposed into their singular vectors and values within a very short amount of time.

For this particular problem, the use of the quadratic spline regularization leads to a system matrix having a number of interesting properties. Those properties can be seen in the Picard plot of the SVD components as shown in Fig. 3.

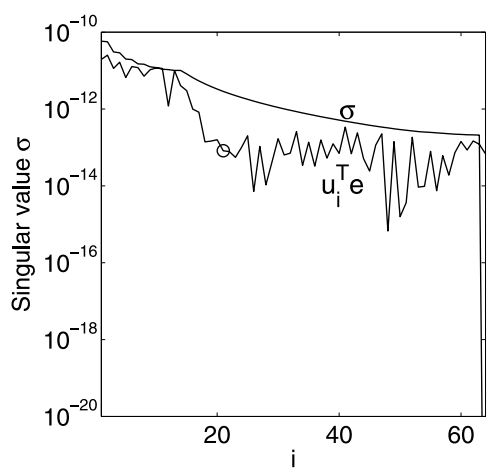

Fig. 3 Picard plot of SDOF problem with quadratic spline approximation.

As can be seen in the figure, the singular value $\sigma$ decay continuously until the last component. This seems to suggest that the problem is not only an ill-posed problem, but also a rank deficient problem. When the last singular value is removed, the problem becomes an ill-posed problem.

The Picard plot in Fig. 3 suggests that since $u_{i}^{T} e$ decays faster than $\sigma_{i}$, then it is possible to regularize the solution by filtering out the small singular values. The figure also suggests that the components of $u_{i}^{T} e$ level off initially at $i=21$.

In Fig. 4, we construct the L-curve that computed by varying the regularization parameter $k$ from 1 to 63 excluding the last singular value. The corner of the L-curve, shown as a circle, is obtained at $k=21$. The solution, shown in Fig. 2, is the most optimal solution where provides the best fitness to the data while maintaining a small influence of the noise.

Since the exact solution, for this particular example, is available, we can easily compute the relative estimation error of the best solution; the result is $4.7 \%$.

\subsection{Impact of a Sphere onto a Beam: Finite Element Analysis}

In this section, the regularized quadratic spline approximation method is used to estimate the impact-force occurring on impact of a sphere onto a beam. The problem takes into account the nonlinearity due the change of the contact condition along the loading.

The both beam and sphere are made of steel material having a Young modulus of $210 \mathrm{GPa}$, a Poisson ratio of 0.3 , and a density of $7.8 \times 10^{-6} \mathrm{~kg} / \mathrm{mm}^{3}$. The beam is $350 \mathrm{~mm}$ long, $25 \mathrm{~mm}$ 


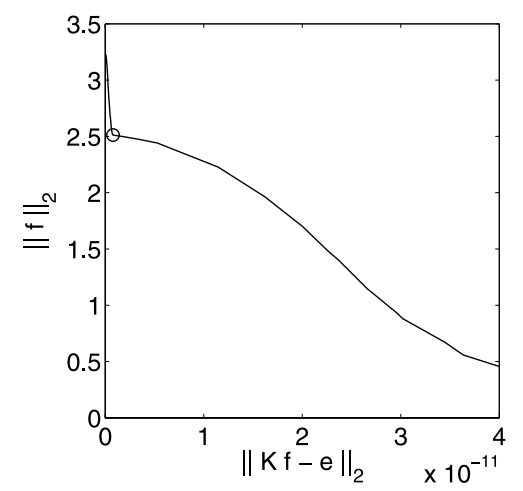

Fig. 4 The L-curve of the SDOF problem.

wide, and $15 \mathrm{~mm}$ thick. The sphere is $18 \mathrm{~mm}$ diametric.

Similar to the previous example problem, the impulse response function has to be determined prior performing the inverse analysis.

By use the Hertz theory of contact and the Euler-Bernoulli beam theory, Goldsmith ${ }^{(8)}$ derived the governing equation that relates the impact-force $f(t)$ to the lateral displacement $w(x, t)$ :

$$
w(x, t)=\frac{1}{\rho A} \sum_{i=1}^{\infty} \frac{\left(X_{i}(x)\right)^{2}}{\omega_{i} \int_{0}^{L} X_{i}^{2} d x} \int_{0}^{t} f(\tau) \sin \omega_{i}(t-\tau) d \tau,
$$

where $\rho$ is the density, $A$ is the cross-section area, $L$ is the beam length, $\omega_{i}$ is the beam natural frequencies, and $X_{i}$ is the eigenfunctions.

For the case of a beam without any support at its both ends, the natural frequency can be obtained by, firstly, solving a stiff nonlinear equation:

$$
\cos \left(\xi_{i} L\right) \cdot \cosh \left(\xi_{i} L\right)=1,
$$

for $\xi_{i}$, and then, solving

$$
\omega_{i}=\xi_{i}^{2} \sqrt{\frac{E I}{\rho A}} .
$$

The symbol $E$ denotes the Young modulus, and $I$ denotes the moment of inertia about the neutral axis.

The eigenfunction for the beam that free at both ends is

$$
X_{i}(x)=\frac{\cosh \xi_{i} x+\cos \xi_{i} x}{\cosh \xi_{i} L-\cos \xi_{i} L}-\frac{\sinh \xi_{i} x+\sin \xi_{i} x}{\sinh \xi_{i} L-\sin \xi_{i} L} .
$$

The exact impulse response function can be obtained from Eq. (28) by substituting $f(t)$ with the unit impulse function and substituting $X_{i}(x)$ with Eq. (31).

This section presents a numerical approach that use to obtain the impulse response function. This approach is taken because the above exact solution provides a less accurate beam response, particularly, along the beam transient response. This is due to the fact that the above solution is derived without taking into account the wave propagation phenomena.

To numerically determine the impulse response function, firstly, we generalized Eq. (28):

$$
h(t)=\sum_{i=1}^{N} a_{i} \sin \omega_{i} t,
$$

and numerically performed the modal analysis by means the finite element method to obtain the beam natural frequencies. Table 1 presents the results of the first nine frequencies.

And finally, we sequentially determine the constants $a_{i}$ by the least-squares method. Table 2 presents the pseudo-code that used to determine the constants $a_{i}$; the results, for $N=4$, are presented in Table 3. 
Table 1 The first nine natural frequencies of the beam in $\mathrm{kHz}$

\begin{tabular}{lcccc}
\hline 1.0692 & 5.3723 & 7.4106 & 11.962 & 19.836 \\
22.185 & 28.37 & 36.805 & 37.20 & - \\
\hline
\end{tabular}

Table 2 The pseudo-code of the sequential least-squares method.

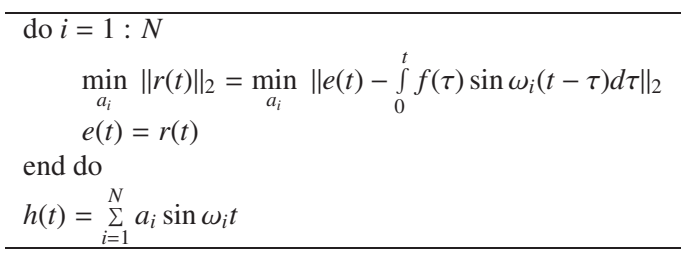

Table 3 The constants of the impulse response function

$$
\begin{aligned}
& a_{1}=4.3467245 \mathrm{e}-006 \\
& a_{2}=4.2513664 \mathrm{e}-006 \\
& a_{3}=4.0393526 \mathrm{e}-007 \\
& a_{4}=4.3723226 \mathrm{e}-006
\end{aligned}
$$

The numerically constructed impulse response function, presented in Fig. 5, is verified before it is being use in the inverse analysis. Figure 6 shows the strain at the beam obtained by the finite element analysis and that obtained by use the constructed impulse response function. The finite element analysis is performed by use a mesh presented in Figure 7. The analysis is

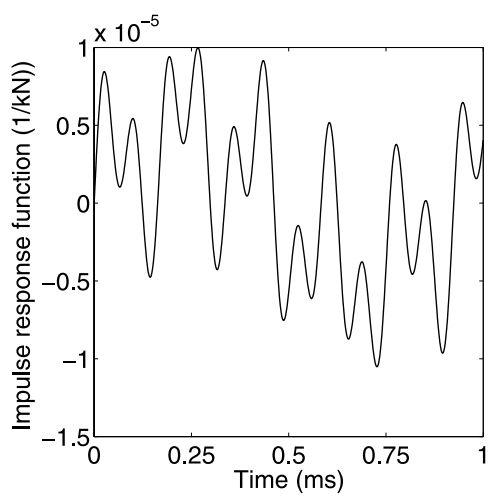

Fig. 5 The impulse response function of the beam for the high speed impact case.

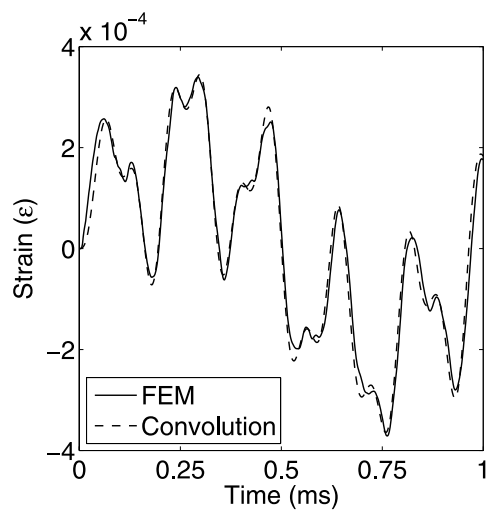

Fig. 6 The beam responses by the finite element method and by use the approximated impulse response function.

performed by use LS-DYNA. The sphere impacted the beam at a velocity of $10 \mathrm{~mm} / \mathrm{ms}$. The both sphere and beam are assumed to be made of the same material having Young modulus of $210 \mathrm{GPa}$ and Poisson ratio of 0.3 . It is also assumed that the both objects deform elastically. 
The response by the finite element method presented in Fig. 6 is the strain data in $x$ direction at the bottom of the beam which is indicated as the measurement point in Fig. 7.

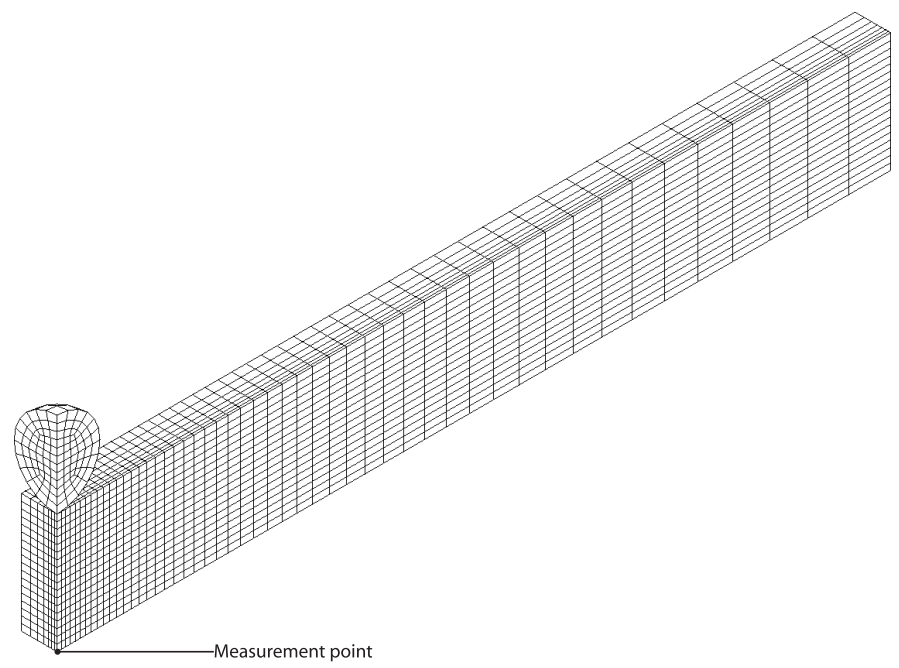

Fig. 7 Finite element mesh of the impact of a sphere and a beam

For this particular problem, the singular value decomposition analysis also reveals the same phenomena as those for the SDOF case. The last eigenvalue is suddenly dropped, and when the value is removed, the plot of the residual norm, $\|K f-e\|$, and the solution norm, $\|f\|$, by changing the regularization parameter from 1 to 63 forms an L-shape curve (see Fig. 9). At the curve corner, the optimal regularization parameter is obtained at $k=13$, which corresponds to the optimal impact-force depicted in Fig. 8.

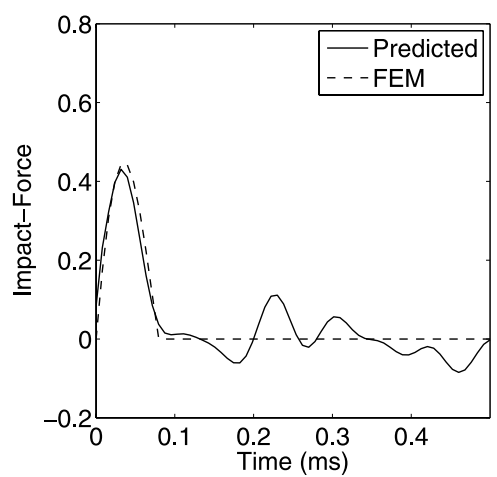

Fig. 8 The estimated impact-force for the case of the sphere-beam impact where the data are obtained from the finite element analysis.

The finite element analysis presented above does not only provide the strain at the measurement point, but also the data of the forces occurred on the interface beam-sphere. The sum of those interface forces is plotted as the dashed line in Fig. 8.

Therefore, it is concluded that the regularized quadratic spline approximation method provides a good solution, in particular, along the loading segment of the impact-force profile.

\subsection{Impact of a Sphere onto a Beam: Experiment}

As mentioned in Section 1, an ill-posed system equation magnifies the data error, and then produces an oscillating solution. A linear perturbation analysis suggests that the error in the solution is proportional to magnitude of the condition number ${ }^{(13)}$. For the present case, with the condition number in order of $1 \times 10^{18}$, an excessive oscillation can be expected to appear in the solution without regularization. 


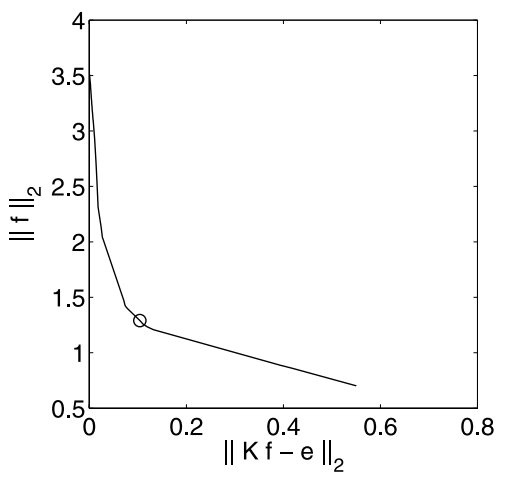

Fig. 9 The L-curve for the sphere-beam impact problem.

In this section, we construct the impact-force based on the data obtained from an impact experiment. The study is necessary because the experimental data, especially those from a high-speed impact experiment, are having a rather big discrepancy to compare with the data of the finite element analysis and of the analytical function.

An impact event similar to that depicted in Fig. 7 is reproduced in the experiment. The beam is supported by four light strings attached the beam ends, and a gas gun system is used to launch the sphere. Ten impacts are performed at a constant gas pressure. The results, the strain time history at the center of the back surface, of those ten impacts are quite identical. One of the results is reproduced in Fig. 10 as the solid line.

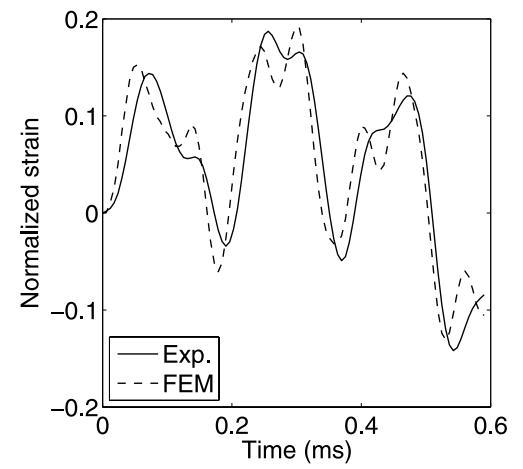

Fig. 10 The 2-norm normalized strain time histories obtained in the impact experiment and in the finite element analysis.

In Fig. 10, in addition to the experimental data, the strain time history obtained from the finite element analysis is also shown as the broken line. This result is obtained by adjusting the sphere velocity in the finite element analysis until the strain time history best matches the strain from the experiment. The analysis is required to obtain the impact-force that will be used to measure the accuracy of the inverse analysis.

The estimated impact-force by the inverse analysis is shown in Fig. 11 besides the impactforce obtained from the finite element analysis. The solution is obtained at regularization parameter $k$ of 11 . The singular value analysis also reveals the same behavior as those appeared in the previous two cases. The last component of the singular values also needs to be removed to construct the L-curve.

The estimated impact-force slightly shifts to the right of the force obtained by the finite element analysis. This result can be understood because the data that used to reconstruct the force also shift to the right of the strain of the finite element analysis. Nevertheless, this last example problem suggests that the quadratic spline method provides an acceptable estimated impact-force although its based on the data that significantly diverge from the ideal data because of the noise. 


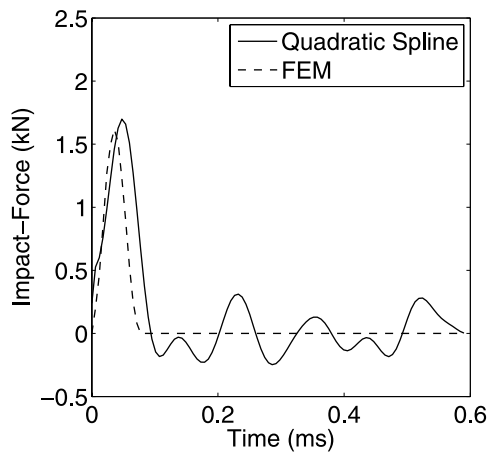

Fig. 11 The estimated impact-force for data obtained in the impact experiment.

\section{Discussions}

The singular value decomposition is the most suitable method to solve the ill-posed inverse problem ${ }^{(1)}$ because its allows us to obtain information of the spectra that affected by the data noise; thus, degree of the regularization can be easily determined to fairly reconstruct the solution. However, the method requires a high computation cost; the highest among the direct solution methods ${ }^{(14)}$. Therefore, it is only feasible for the small scale inverse problem. For the large scale inverse problem, the quadratic spline can be easily reconstructed to approximate the problem and to reduce the problem size to a size that can be handled with the singular value decomposition method.

\section{Conclusions}

This research on the impact-force reconstruction has shown that the quadratic spline approximation with conjunction with the truncated singular value decomposition method is able to provide a quite accurate solution even thought the analysis is based on a highly contaminated data. Current finding suggests that the L-curve can only be accurately constructed when the last singular value component is excluded.

However the quadratic spline that constructed by use an uniform distribution of the knots produces oscillating solution in the unloading region. This suggests that the knots number on the unloading part should be reduced. Therefore, a new approach that based on an the variable segment length has to be developed.

\section{References}

( 1 ) P. C. Hansen. Rank Deficient and Discrete Ill Posed Problems. SIAM Monograph on Mathematical Modeling and Computation. SIAM, Philadelphia, 1998.

( 2 ) A. N. Tikhonov and V. Y. Arsenin. Solution of Ill-Posed Problems. John Wiley \& Sons, New York, 1977.

( 3 ) X. Q. Zhu and S. S. Law. Orthogonal function in moving loads identification on a multispan bridge. Journal of Sound and Vibration, 245(2):329-345, August 2001.

( 4 ) Bor Tsuen Wang and Chun Shien Chiu. Determination of unknown impact force ating on a simple supported beam. Mechanical systems and signal processing, 17(3):683-704, 2003.

( 5 ) E. Jacquelin, A. Bennani, and P. Hamelin. Force reconstruction: analysis and regularization of a deconvolution problem. Journal of Sound and Vibration, 265:81-107, 2003.

(6) Yi Liu and W. Steve Shepard Jr. Dynamic force identification based on enhanced least squares and total least-squares schemes in the frequency domain. Journal of sound and vibration, 282:37-60, 2005.

( 7 ) Adam A. Cardi, Douglas E. Adams, and Shawn Walsh. Ceramic body armor single 
impact force identification on a compliant torso using acceleration response mapping. Structural health monitoring, 5(4):355-372, 2006.

( 8 ) Werner Goldsmith. Impact-The Theory and Physical Behaviour of Colliding Solids. Dover publications, Inc., 2001.

( 9 ) Fergyanto E. Gunawan, Hiroomi Homma, and Yasuhiro Kanto. Two-step b-splines regularization method for solving an ill-posed problem of impact-force reconstruction. Journal of Sound Vibration, 297:200-214, Oct 2006.

(10) V. I. Dmitriev and Zh. Ingtem. Solving an integral equation of the first kind by spline approximation. Computational mathematics and modeling, 15:99-104, 2004.

(11) Ljung Lennart. System Identification-Theory for The User. Prentice-Hall, Inc., Englewood Cliffs, New Jersey, 07632, 1987.

(12) Jimin He and Zhi-Fang Hu. Modal Analysis. Butterworth \& Heienmann, 2001.

(13) James W. Demmel. Applied Numerical Linear Algebra. SIAM, 1997.

(14) Gene H. Golub and Charles F. Van Loan. Matrix Computation. The Johns Hopkins University Press, second edition, 1996. 